\title{
Correction to: Psychiatric disorders and SLC6A4 gene variants: possible effects on alcohol dependence and alzheimer's disease
}

\author{
Marco Calabrò ${ }^{1}$ - Laura Mandelli ${ }^{2}$. Concetta Crisafulli ${ }^{1}$ - Stefano Porcelli ${ }^{2}$. Diego Albani ${ }^{3}$ - Antonis Politis ${ }^{4}$. \\ George N. Papadimitriou ${ }^{4}$. Marco Di Nicola ${ }^{5,13}$ - Luigi Janiri ${ }^{5} \cdot$ Roberto Colombo $^{5}$. Giovanni Martinotti ${ }^{6}$. \\ Antonello Bellomo ${ }^{7}$. Eduard Vieta ${ }^{8}$. Stefano Bonassi ${ }^{9}$. Alessandra Frustaci ${ }^{10}$. Giuseppe Ducci ${ }^{11}$. Stefano Landi ${ }^{12}$. \\ Stefania Boccia ${ }^{5,13} \cdot$ Alessandro Serretti ${ }^{2}$
}

Published online: 22 January 2020

(c) Springer Nature B.V. 2020

\section{Correction to: Molecular Biology Reports (2020) 47:191-200 https://doi.org/10.1007/s11033-019-05119-5}

The following authors have double affiliations: Stefania Boccia, Marco Di Nicola and they should read

5. Fondazione Policlinico Universitario A. Gemelli IRCCS, Roma, Italia and

13. Universita' Cattolica del Sacro Cuore, Roma, Italia

Publisher's Note Springer Nature remains neutral with regard to jurisdictional claims in published maps and institutional affiliations.
The original article can be found online at https://doi.org/10.1007/ s11033-019-05119-5.

\section{Alessandro Serretti}

alessandro.serretti@unibo.it

1 Department of Biomedical and Dental Sciences and Morphofunctional Imaging, University of Messina, Messina, Italy

2 Department of Biomedical and NeuroMotor Sciences, University of Bologna, Viale Carlo Pepoli 5, 40123 Bologna, Italy

3 Laboratory of Biology of Neurodegenerative Disorders, Neuroscience Department, Istituto di Ricerche Farmacologiche Mario Negri IRCSS, Milan, Italy

4 Department of Psychiatry, University of Athens Medical School, Eginition Hospital, Athens, Greece

5 Fondazione Policlinico Universitario “A. Gemelli” - IRCCS, Università Cattolica del Sacro Cuore, Rome, Italy

6 Department of Neuroscience, Imaging and Clinical Sciences, University "G. d'Annunzio" of Chieti, Chieti, Italy
7 Psychiatry Unit, Department of Medical Sciences, University of Foggia, Foggia, Italy

8 Bipolar Disorders Unit, Institute of Neuroscience, Hospital Clínic, University of Barcelona, IDIBAPS, CIBERSAM, Barcelona, Catalonia, Spain

9 Unit of Clinical and Molecular Epidemiology, IRCCS San Raffaele Pisana, Rome, Italy, and Department of Human Sciences and Quality of Life Promotion, San Raffaele University, Rome, Italy

10 Barnet, Enfield and Haringey Mental Health NHS Trust, St.Ann's Hospital, St.Ann's Road, London N15 3 TH, UK

11 Mental Health Department, ASL Roma 1, Rome, Italy

12 Dipartimento di Biologia, Università di Pisa, Pisa, Italy

13 Section of Hygiene, Institute of Public Health, Universita' Cattolica del Sacro Cuore, Fondazione Policlinico "Agostino Gemelli” IRCCS, Rome, Italy 